\title{
A INFLUÊNCIA DAS EMOÇÕES NAS FALSAS MEMÓRIAS: UMA REVISÃO CRÍTICA'
}

\section{Renato Favarin dos Santos Lilian Milnitsky Stein}

Resumo: Neste artigo revisamos estudos relativos à influencia das emoções no funcionamento da memória e, em específico, na produção de falsas memórias. As falsas memórias são um tipo de distorção mnemônica que consistem na recuperação de eventos que nunca ocorreram. As emoções são descritas através das dimensões de valência e alerta. Numerosos estudos têm sugerido que a valência e o alerta beneficiam a memória através de distintos processos cognitivos e mecanismos neurais. Já as pesquisas sobre a influência das emoções na produção de falsas memórias são bastante incipientes, e divergem quanto aos seus resultados. $O$ foco principal será a discussão metodológica de pesquisas que relacionam as emoções aos falsos reconhecimentos. Por fim, apresentamos algumas sugestões para futuros estudos, e o atual estado de pesquisas desenvolvidas no Brasil.

Palavras-chave: Emoção. Memória. Falsas memórias.

\section{Introdução}

Por muito tempo a relação entre a emoção e a cognição não se constituiu um objeto legítimo das ciências da mente, sob a alegação de que ambas se encontravam em pólos opostos da experiência humana (Damásio, 1996). Contudo, recentemente, CAPES. 
esse tema tem atraído um crescente interesse da comunidade científica. Influenciados por um novo Zeitgeist, psicólogos, psiquiatras, neurologistas e biólogos têm proposto novos projetos de pesquisa, que consideram a emoção e a cognição como domínios complementares (Dalgleish, 2004). Dentre as novas questões a serem respondidas, destacamos:como as emoções influenciam o funcionamento da memória?

Para responder a essa pergunta, muitos pesquisadores têm empregado uma variedade de metodologias. Pesquisas em laboratórios têm sugerido que palavras e fotografias com conteúdo emocional são melhor recuperadas em testes de recordação e reconhecimento do que palavras e fotografias sem conteúdo emocional (Buchanan, Denburg, Tranel, \& Adolphs, 2001; Kensinger \& Corkin, 2003; LaBar \& Phelps, 1998; Ochsner, 2000). Adicionalmente, estudos que analisam memórias autobiográficas têm demonstrado que experiências autobiográficas emocionais são melhor recuperadas do que experiências sem conteúdo emocional (Conway et al., 1994; Rubin \& Kozin, 1984). Um exemplo extremo do impacto da emoção na memória autobiográfica é conhecido pela denominação flashbulb memory (memórias de lampejo) (Brown \& Kulick, 1977), que consiste em recordações ricas em detalhes e acompanhadas por altos níveis de confiança subjetiva. Pessoas que apresentam esse tipo de fenômeno, usualmente, referem-se a essas lembranças como filmes que passam em suas cabeças (Schacter, 2001).

Contudo, alguns estudos utilizando memórias autobiográficas têm levantado suspeitas a respeito da exatidão das memórias para eventos emocionais, sugerindo que essas também são suscetíveis a distorções (Levine, 1997; Levine \& Safer, 2002; Talarico \& Rubin, 2003). Essas suspeitas, no entanto, são difíceis de serem testadas com memórias autobiográficas, haja vista que torna difícil, se não impossível, uma comparação minuciosa entre as informações recuperadas e os detalhes do evento original (Berntsen, 2002; Reisberg \& Hertel, 2004).

A fim de sanar essas limitações metodológicas, estudos recentes, utilizando palavras como estímulos, têm investigado a influência das emoções na distorção de memórias, com destaque para a distorção mnemônica, denominada de falsas memórias (Kensinger \& Corkin, 2004a; Maratos, Allan, \& Rugg, 2000; Pesta, Sanders, \& Murphy, 2001; McNeely, Dywan, \& Segalowitz, 2004). Segundo Roediger e McDermott (1995), as falsas memórias são um tipo de distorção mnemônica que consiste na recuperação de eventos que nunca foram vivenciados. Embora a ocorrência de falsas memórias seja, em certa medida, comum em nossas vidas, assim como outras falhas mnemônicas, como o esquecimento, ela tem recebido uma atenção especial por parte da comunidade científica, pois seu estudo gera insights a respeito da natureza reconstrutiva da memória (Schacter \& Slotnick, 2004).

Nessa revisão, contemplaremos estudos realizados em laboratórios que relacionam as emoções ao funcionamento da memória, e mais especificamente à produção de falsas memórias. Iniciaremos apresentando, em 
separado, teorias e pesquisas sobre emoções e falsas memórias. Em seguida, revisaremos achados relativos ao impacto de estímulos emocionais em recuperações verdadeiras (memórias verdadeiras) e falsas (falsas memórias). Depois, apresentaremos uma série de críticas às metodologias utilizadas nessas pesquisas, bem como sugestões para futuros estudos na área.

\section{Emoções}

As emoções podem ser definidas, de modo abrangente, como coleções específicas e consistentes de respostas cognitivas e fisiológicas acionadas por sistemas cerebrais que preparam o organismo para a ação e a interação social (Damásio, 2000; Lang, 1995; Lazarus, 1991). As reações emocionais podem ser mensuradas através de relatos subjetivos (e.g., escalas avaliativas), respostas fisiológicas (e.g., freqüência cardíaca e condutância elétrica da pele) e observação de comportamentos (e.g., expressões faciais) (Lang, 1969). Segundo Bradley e Lang (1994), as experiências humanas podem ser caracterizadas através de duas dimensões afetivas principais, a valência e o alerta. ${ }^{2} \mathrm{~A}$ valência refere-se a um contínuo avaliativo que varia da classificação de desprazer (desagradável) a prazer (agradável), passando pela classificação neutra. Já o alerta refere-se a um contínuo avaliativo que varia da calma à estimulação. A reação emocional a qualquer estímulo (e.g., imagens e palavras) pode ser classificada quanto à valência e quanto ao alerta.Com o propósito de possibilitar a avaliação afetiva de estímulos, para serem utilizados em estudos sobre motivação, atenção e memória, Lang (1980) desenvolveu uma medida pictográfica não verbal para a avaliação subjetiva da valência e do alerta, o Self-Assessment Manikin (SAM). Usualmente, estímulos que provocam reações emocionais classificadas, através do SAM, com níveis baixos de valência são descritos como negativos, com níveis médios como neutros, e com níveis altos como positivos. Quanto ao alerta, os estímulos classificados com níveis baixos são descritos como não estimulantes, e com níveis altos como estimulantes.

As dimensões valência e alerta constituem conjuntamente dois sistemas motivacionais específicos, conhecidos como sistemas apetitivo e aversivo. $\mathrm{O}$ sistema apetitivo é representado por comportamentos de aproximação e o sistema aversivo é representado por comportamentos de evitação e fuga. Enquanto a resposta de valência dirige o comportamento ativando o sistema motivacional (apetitivo ou aversivo), a resposta de alerta corresponde à magnitude dessa reposta (Lang, 1995). 


\section{Falsas memórias}

Tradicionalmente, as falsas memórias vêm sendo investigadas através de vários tipos de procedimentos experimentais que potencializam sua ocorrência, utilizando materiais, como: seqüências de slides (Loftus, Miller, \& Burns, 1978), vídeo-tapes (Loftus \& Palmer, 1974) e sentenças (Bransford \& Franks, 1971). Na última década, uma metodologia bastante difundida é o da lista de palavras associadas. Esse procedimento, conhecido pela sigla DRM, foi desenvolvido por Roediger e McDermott (1995), baseado no trabalho de Deese (1959). O DRM é constituído por listas de palavras que são apresentadas para serem memorizadas. As palavras de cada lista giram em torno de um mesmo tema (e.g., pátria, símbolo, nação, mastro, país, verde, pano, hino, flâmula, honra, identificação, representação, amarelo, haste e estado versam sobre o tema bandeira). A palavra crítica -bandeira-, que traduz a essência temática da lista e que está semanticamente associada a todas as outras palavras da mesma, não é apresentada na etapa de memorização. $\mathrm{O}$ efeito consistente observado é que, quando testada a memória para a lista original, a palavra bandeira é recordada ou reconhecida muitas vezes na mesma proporção que palavras da lista estudada (Stein \& Pergher, 2001). Outras versões do DRM foram construídas com listas de palavras associadas ortograficamente (Schacter, Verfaellie, \& Anes, 1997; Watson, Balota, \& Roediger, 2003) e foneticamente (Sommers \& Lewis, 1999), também obtendo êxito na produção de falsas memórias.

Desde que Roediger e McDermott (1995) desenvolveram o procedimento DRM, muitas teorias vêm sendo propostas para explicar o fenômeno das falsas memórias (para uma revisão sobre o assunto, ver Brainerd e Reyna, 2005, cap. 3). Por exemplo, a teoria da ativação/monitoramento da fonte sugere que a apresentação de palavras de uma mesma lista durante a fase de estudo ativa a palavra crítica na memória semântica. E, posteriormente, no momento do teste, essa palavra pode ser atribuída erroneamente (falha no monitoramento) às listas apresentadas (Roediger, Watson, McDermott, \& Gallo, 2001). Outra teoria bastante utilizada é a teoria do traço difuso (TTD), que propõe que a memória não é um sistema unitário, mas sim constituído de dois sistemas independentes e em paralelo (Reyna \& Brainerd, 1995). Esses dois sistemas codificam as informações sob a forma de diferentes representações, denominadas representações literais e de essência. A memória literal armazena os traços específicos, episódicos e detalhes das palavras, enquanto a memória de essência armazena o sentido de forma inespecífica, ou seja, o significado, e os padrões gerais das palavras apresentadas. Para a TTD, as falsas memórias seriam decorrentes da recuperação de memórias da essência do material estudado, quando as memórias literais não estão mais acessíveis. Já as memórias verdadeiras seriam decorrentes, em sua maior parte, da recuperação de memórias literais (Brainerd \& Reyna, 2002). 


\section{Emoções e memória}

Várias pesquisas têm mostrado a influência das emoções na memória. Palavras e fotografias classificadas como sendo de valência negativa e não estimulantes (Kensinger \& Corkin, 2003; Ochsner, 2000) e palavras e fotografias classificadas como sendo de valência (negativa ou positiva) e estimulantes (Cahill \& McGaugh, 1998; Hamann, Ely, Grafton, \& Kilts, 1999) possuem maior probabilidade de serem recuperadas corretamente, em comparação a estímulos semelhantes classificados como neutros e não estimulantes. A classificação dos estímulos nessas diferentes dimensões emocionais é necessária, pois alguns estudos têm mostrado que a valência e o alerta influenciam os índices de recuperações verdadeiras através de diferentes mecanismos neurais e processos cognitivos (para uma revisão sobre o assunto, ver Kensinger, 2004).

Pesquisas utilizando recursos de neuroimagem têm revelado que a ação da valência está relacionada à ativação de porções laterais e mediais do córtex pré-frontal e do hipocampo (Dolcos, LaBar, \& Cabeza, 2004; Paller \&Wagner, 2002). A vantagem na recuperação de itens (e.g., palavras e fotografias) que são caracterizados como sendo de valência emocional, tanto negativa quanto positiva, parece ser explicada por processos cognitivos de elaboração que atuam na fase de codificação (Craik, 2002). O processo de elaboração consiste no estabelecimento de conexões, realizadas de maneira consciente, entre os estímulos apresentados e os conhecimentos prévios já armazenados na memória. A elaboração pode ocorrer através de duas formas: elaboração semântica e elaboração autobiográfica. A elaboração semântica consiste em construções de redes de significados entre os itens codificados. Já a elaboração autobiográfica ocorre quando os estímulos codificados são associados a experiências vividas previamente (Doerksen \& Shimamura, 2002). Segundo a hipótese da elaboração autobiográfica, diante da apresentação de itens (e.g., palavras e fotografias) classificados como sendo de valência neutra e valência emocional (positiva ou negativa), é mais provável que as pessoas associem os estímulos emocionais às suas experiências autobiográficas, em comparação aos neutros (Kensinger, 2004). Reforçando o papel da atenção nesses processos de elaboração, Kensinger e Corkin (2004b) mostraram que a realização de tarefas que dividem a atenção (e.g., discriminação de melodias) durante a codificação de palavras classificadas como sendo de valência negativa e não alerta anulam o benefício da valência nos índices de recuperação verdadeira.

Quanto à ação do alerta, estudos de neuroimagem têm revelado que ele está estreitamente relacionado à ativação da amídala e sua relação com o hipocampo e o córtex pré-frontal (e.g., Dolcos et al., 2004). Corroborando esses achados, algumas pesquisas têm sugerido que pessoas com lesões na amídala não apresentam os benefícios do alerta emocional nos índices de recuperações verdadeiras de palavras e narrativas acompanhadas de 
fotografias (Adolphs, Cahill, Schul, \& Babinsky, 1997; Adolphs, Tranel, \& Denburg, 2000).

A ação do alerta na memória caracteriza-se por ocorrer de maneira automática, não intencional, e se dá durante as fases de codificação e consolidação. Na codificação, o alerta produzido por um estímulo (por exemplo, a palavra morte ou amor) faz com que haja a modulação do nível de atenção, tornando a sua recuperação mais provável (Kensinger, 2004). Segundo Hamann et al. (1999), a ação do alerta é independente da valência, ocorrendo tanto frente a itens classificados como negativos quanto positivos.

Adicionalmente, alguns estudos têm sugerido que o alerta reforça a codificação de aspectos centrais do estímulo através de mecanismos de atenção não intencionais, ao mesmo tempo em que tende a diminuir a codificação de detalhes periféricos dos estímulos (Burke, Heuer, \& Reisberg, 1992; Christianson \& Loftus, 1991). Por exemplo, diante da apresentação de uma foto de um acidente automobilístico entre dois carros em uma rodovia, as pessoas tendem a recuperar mais os aspectos centrais e significativos do evento (e.g., os carros amassados) do que aspectos periféricos do evento (e.g., uma placa de trânsito ou outdoors de propaganda no acostamento) (para uma revisão sobre o assunto, ver Reisberg e Hertel, 2004, cap. 1).

Esse padrão de ação do alerta na memória também vem sendo encontrado em estudos sobre testemunhos oculares de crimes, recebendo a denominação de weapon-focus (foco na arma) (Loftus, 1979). Esse fenômeno consiste na maior recuperação de detalhes referentes à arma utilizada em um assalto em comparação a outros aspectos do evento. Dessa forma, diante de um assalto a mão armada, as vítimas tendem a evocar com maior exatidão os detalhes da arma utilizada no crime do que outras informações sobre o evento (e.g., a cor da roupa utilizada pelo assaltante). Corroborando a hipótese de que a ação do alerta ocorre independente da atenção consciente, Kensinger e Corkin (2004b) mostraram que o efeito do alerta na memória, durante a codificação de palavras classificadas como sendo de valência negativa e estimulantes, não é anulado por procedimentos que dividem a atenção do participante no momento da codificação desses itens.

No momento da consolidação da memória, os hormônios liberados pela amídala na fase da codificação agem no hipocampo, auxiliando o armazenamento dos estímulos e tornando-os mais resistentes ao esquecimento e a interferências, facilitando assim sua recuperação (MacGaugh, 2000). Corroborando esse achado, várias pesquisas têm sugerido que as vantagens nas recuperações de itens classificados como estimulantes, em comparação a itens não estimulantes, são maiores quando a testagem ocorre após intervalos que variam de 1 a 24 horas (tempo necessário para haver a consolidação), em comparação à testagem imediata (e.g. LaBar \& Phelps, 1998). 


\section{Emoções e falsas memórias}

Desde a década passada, pesquisas realizadas na área da saúde mental e na área jurídica têm sugerido que as emoções podem influenciar a produção de falsas memórias. Na área da saúde mental, o fenômeno das falsas memórias vem chamando atenção, pois alguns estudos têm relatado que determinadas técnicas psicoterápicas, que se baseiam na recuperação de memórias emocionais da infância, podem produzir lembranças vívidas de eventos que na realidade não ocorreram, por exemplo, supostos casos de violência sexual sofrida na infância (Lindsay, 1994). Na área jurídica, o impacto da emoção no funcionamento da memória pode comprometer o exercício da justiça, visto que a pessoa que presenciou algum crime, infração e/ou foi alvo de violência pode estar sujeita a distorções de suas memórias (Eisen, Quas, \& Goodman, 2002).

Recentemente, estão sendo desenvolvidas pesquisas em laboratórios que avaliam a influência das emoções na produção de falsas memórias. Esses estudos têm utilizado, em sua grande maioria, testes de reconhecimento, negligenciando a influência das emoções na produção de falsas recordações (McNeely et al., 2004). Tendo em vista isso, na presente revisão, daqui por diante, utilizaremos o termo falsas memórias como sinônimo de falsos reconhecimentos. Os estudos sobre falsas memórias e emoções podem ser divididos em dois grupos: (1) os que apresentam estímulos emocionais durante a fase de estudo e durante a fase de teste, e (2) os que apresentam estímulos emocionais somente na fase de teste.

\section{Estímulos emocionais na fase de estudo e de teste}

Com o propósito de analisar a influência das emoções no reconhecimento verdadeiro de palavras e na produção de falsas memórias, em uma amostra de estudantes universitários, Maratos et al. (2000) realizaram uma pesquisa que utilizou palavras de valência neutra (e.g., cadeira) e valência negativa (e.g., medo). Imediatamente após a apresentação dos itens, os participantes realizaram um teste de reconhecimento. Os reconhecimentos verdadeiros constituíram na aceitação de palavras neutras e negativas apresentadas na fase de estudo, enquanto, as falsas memórias, na aceitação de palavras novas neutras e negativas. Os resultados obtidos foram que $o$ índice de reconhecimento verdadeiro de itens negativos foi superior ao encontrado para os itens neutros. Além disso, o índice de falsas memórias de itens negativos foi o dobro daquele para itens neutros.

Esses últimos resultados foram corroborados por medidas eletrofisiológicas realizadas durante o teste de reconhecimento, através de potenciais relacionados ao evento (PRE). Houve diferenças significativas entre a 
atividade elétrica do cérebro durante a codificação de palavras novas negativas, em comparação a atividade elétrica mensurada na codificação de palavras novas neutras. Os pesquisadores sugeriram que esses resultados (comportamentais e eletrofisiológicos) foram decorrentes da associação semântica compartilhada entre as palavras emocionais, e não de sua carga emocional propriamente dita. Pois alguns estudos sugerem que a codificação de palavras novas neutras associadas semanticamente a palavras apresentadas na fase de estudo é acompanhada de um padrão eletrofisiológico semelhante ao encontrado na codificação de palavras novas negativas no momento do teste de reconhecimento (Bentin, McCarthy, \& Wood, 1985). Por conseguinte, Maratos et al. (2000) sugeriram que os altos índices de falsos reconhecimentos de itens negativos não seriam resultantes da carga emocional, e sim da maior associação semântica existente entre as palavras negativas apresentadas na fase de estudo e as palavras negativas novas apresentadas somente na fase de teste, em comparação com as palavras neutras.

McNeely et al. (2004) realizaram dois experimentos com universitários para verificar a influência das emoções no reconhecimento verdadeiro e falso de palavras. No primeiro experimento, os pesquisadores replicaram o estudo de Maratos et al. (2000), encontrando resultados semelhantes. Ou seja, tanto os índices de reconhecimento verdadeiro quanto falso para palavras negativas foram superiores àqueles encontrados para as palavras neutras. No entanto, McNeely et al. questionaram a hipótese, apresentada por Maratos et al., de que o alto índice de falsas memórias emocionais ocorreria em conseqüência de uma suposta associação semântica existente entre as palavras negativas, pois nesses estudos não foram utilizadas palavras neutras associadas semanticamente para servir como uma medida de comparação entre as forças associativas dos conjuntos de palavras neutras e emocionais.

Com o objetivo de verificar a influência da associação semântica na produção de falsas memórias emocionais, McNeely et al. (2004) realizaram um segundo experimento. Na fase de estudo foram apresentados os seguintes conjuntos de palavras: a) palavras de valência neutra; b) palavras de valência neutra e fortemente associadas entre si (associação semântica); c) palavras de valência negativa. Após a apresentação dos itens, os participantes realizaram um teste de reconhecimento. $O$ teste de reconhecimento foi composto de: palavras neutras e negativas apresentadas na fase de estudo; palavras novas neutras não associadas semanticamente às apresentadas; palavras novas neutras e associadas semanticamente às apresentadas; e palavras novas negativas. Os reconhecimentos verdadeiros constituíam na aceitação de itens neutros e negativos apresentados na fase de estudo, enquanto, as falsas memórias, na aceitação de itens novos neutros (associados e não associados) e negativos. $O$ índice de reconhecimentos verdadeiros de palavras negativas foi superior àquele encontrado para as 
neutras. Já a taxa de falsas memórias produzida para palavras negativas foi superior tanto à taxa produzida por palavras neutras sem associação semântica quanto por palavras neutras associadas semanticamente. Com base nesses resultados, os pesquisadores sugeriram que a valência emocional negativa influenciaria as taxas de falsas memórias independentemente da associação semântica.

Para avaliar a influência da valência negativa no momento da recuperação, McNeely et al. (2004) realizaram mensurações eletrofisiológicas através de potenciais relacionados ao evento (PRE) durante o teste de reconhecimento. Os pesquisadores identificaram padrões de atividade elétrica distintos durante a leitura de palavras novas negativas em comparação aos padrões elétricos eliciados nas leituras de palavras novas neutras associadas semanticamente e não associadas semanticamente. Dessa forma, eles sugeriram que essa atividade elétrica diferenciada, assim como a superioridade nas taxas de falso reconhecimento, foi decorrente da carga emocional das palavras, e não da associação semântica, tal como foi proposto por Maratos et al. (2000).

Para Mcneely et al.(2004), as diferenças nos índices de falsos reconhecimentos entre palavras neutras e negativas ocorreriam porque os itens negativos, apresentados somente no teste, seriam mais rapidamente codificados do que os itens novos neutros. Segundo os pesquisadores, a maior rapidez na codificação de palavras negativas poderia ser erroneamente interpretada pela mente como um indicador de familiaridade, endossando a aceitação de itens negativos apresentados na fase de estudo e itens novos negativos. Conseqüentemente, isso acarretaria um aumento de reconhecimentos verdadeiros, mas também de falsos reconhecimentos. Para os pesquisadores, esse padrão de funcionamento da mente diante de estímulos negativos poderia ser explicado por um mecanismo engendrado pela evolução para nos tornar mais adaptados ao meio ambiente. Segundo essa hipótese evolucionista, nossa espécie é programada a ser mais responsiva a estímulos negativos, sejam reais (e.g., o animal cobra) ou simbólicos (e.g., a palavra cobra), que poderiam causar-nos algum dano. Assim, o mesmo mecanismo que sustenta os altos índices de reconhecimentos verdadeiros também eleva os índices de falsas memórias.

\section{Estímulos emocionais somente no momento do teste}

Algumas pesquisas realizadas para avaliar a influência das emoções na produção de falsas memórias não apresentam estímulos emocionais durante a fase de estudo, mas somente na fase de teste. O Experimento 1, realizado por Pesta et al. (2001), utilizando uma amostra de estudantes universitários, buscou verificar se palavras classificadas como sendo emocionais (palavras tabu, e.g., rape) poderiam ser falsamente reconhecidas. Para 
tanto, Pesta et al. utilizaram uma versão do DRM composta por seis listas de dez palavras neutras associadas ortograficamente a uma palavra não apresentada na fase de estudo. Do total de seis listas, três eram neutras (e.g., beach, leach, teach, reach, poach, peack, pearch, peace, perch, peal) associadas ortograficamente a uma palavra neutra (peach), e outras três também eram neutras (e.g., cape, nape, tape, ripe, rope, race, rapt, rake, rare, raze), mas associadas a uma palavra emocional (rape). $O$ teste de reconhecimento foi composto por palavras apresentadas durante a fase de estudo, por três novas palavras neutras e por três novas palavras emocionais ortograficamente associadas às apresentadas na fase de estudo. A ocorrência de falsas memórias foi caracterizada pelo reconhecimento de itens não apresentados na fase de estudo. Esse procedimento produziu índices superiores de falso reconhecimento de palavras neutras em comparação às emocionais.

Para Pesta et al. (2001), uma possível hipótese explicativa para esses resultados seria a de que as palavras emocionais são mais distintivas do que as palavras neutras, ou seja, mais singulares, tornando a rejeição desses itens mais fácil. Essa explicação é conhecida como a hipótese da distintividade. Para testá-la, os pesquisadores realizaram um segundo experimento, no qual foi utilizada a mesma metodologia, no entanto foram acrescentadas três palavras emocionais às palavras apresentadas na fase de estudo, com a intenção de diminuir a distintividade dos itens emocionais. Os resultados encontrados corroboraram a hipótese da distintividade, já que o índice de falso reconhecimento de itens emocionais dobrou, no entanto, permaneceu, ainda, menor do que a taxa de falso reconhecimento de itens neutros.

Utilizando o mesmo método desenvolvido por Pesta et al. (2001), Kensinger e Corkin (2004a) avaliaram o efeito das emoções na produção de falsas memórias em amostras de adultos jovens e idosos. Assim como no estudo de Pesta et al., os índices de falsas memórias de itens neutros foram superiores aos encontrados para itens emocionais, para ambas as amostras. Segundo as pesquisadoras, isso ocorreu porque tanto os adultos jovens quanto idosos beneficiam-se da distintividade dos itens emocionais apresentados no teste, permitindo que rejeitassem as palavras emocionais.

Kensinger e Corkin (2004a) sugeriram que a ação da distintividade emocional pode ocorrer de duas formas. A emocionalidade de uma palavra pode ser utilizada como um critério de exclusão, ou seja, ao ler uma palavra emocional no teste de reconhecimento ela é corretamente rejeitada por ser incongruente com as palavras neutras apresentadas na fase de estudo. No entanto, as pesquisadoras questionam se esse efeito ocorre devido à distintividade emocional (de natureza perceptiva) ou à distintividade conceitual (de natureza semântica) entre os itens neutros estudados e os itens emocionais do teste, pois as palavras apresentadas na fase de estudo eram todas neutras. Ainda segundo Kensinger e Corkin (2004a), a distintividade emocional pode também influenciar os índices de falsas memórias através da adoção da heurística da distintividade. De acordo com a heurística 
da distintividade, quanto mais características singulares um determinado estímulo possuir, mais específica é sua codificação e mais criteriosamente se dá o processo de reconhecimento (Schacter, Israel, \& Racine, 1999). Desse modo, diante de uma palavra emocional apresentada no momento do teste, o participante a rejeita, pois ele julga que um item emocional teria chamado sua atenção e, conseqüentemente, teria sido codificado de uma maneira bastante específica na fase de estudo. Por exemplo, ao ler uma palavra emocional apresentada na fase de teste (e.g., estupro), o participante pode utilizar a heurística da distintividade ao pensar (e.g., "Se eu tivesse estudado a palavra "estupro", com certeza eu a lembraria"), o que é menos provável de ocorrer frente a uma nova palavra neutra (e.g., folha).

\section{Considerações Finais}

Nesse artigo, revisamos pesquisas que avaliam a influência das emoções no funcionamento da memória,e em específico na produção de falsas memórias. Por se tratar de um tópico de investigação recente, encontramos poucos estudos na literatura especializada. No entanto, conseguimos identificar alguns padrões metodológicos que permitiram a classificação dessas pesquisas em dois grupos, os que apresentam estímulos emocionais na fase de estudo e na fase de teste e os que apresentam estímulos emocionais somente na fase de teste. Constatamos que esses grupos divergem quanto aos seus resultados. Enquanto as pesquisas que apresentam itens emocionais na fase de estudo e na fase de teste obtêm índices de falsas memórias emocionais superiores aos encontrados para itens neutros, as pesquisas que apresentam itens emocionais somente na fase de teste mostram resultados contrários. Acreditamos que as discrepâncias entre os resultados encontrados e suas subseqüentes hipóteses explicativas se devem não somente às evidentes diferenças metodológicas, mas, sim, a uma série de aspectos negligenciados nessas pesquisas. A seguir, discutiremos os estudos acima citados e apresentaremos uma série de sugestões para futuras pesquisas na área.

Embora Maratos et al. (2000) tenham sugerido que os índices superiores de reconhecimentos verdadeiros e falsos encontrados para as palavras negativas, em comparação às palavras neutras não foram resultantes do impacto da emoção na memória, mas sim da força associativa existente entre as palavras negativas apresentadas na fase de estudo com aquelas novas no teste. Esses pesquisadores não controlaram a força associativa entre as palavras apresentadas na fase de estudo e as apresentadas na fase de teste, tornando difícil a sustentação dessa hipótese.

McNeely et al. (2004) realizaram dois estudos para avaliar a influência das emoções no reconhecimento verdadeiro e falso de palavras. O primeiro estudo utilizou o mesmo método empregado por Maratos et al. (2000), 
e foram encontrados resultados semelhantes. Já no segundo experimento, os pesquisadores procuraram verificar a hipótese sugerida por Maratos et al. com a inclusão de um conjunto de palavras neutras associadas semanticamente. No entanto, assim como Maratos e colaboradores, McNeely et al. não controlaram eventuais diferenças das associações semânticas existentes entre os conjuntos de palavras neutras associadas e negativas utilizadas na fase de estudo com as palavras utilizadas na fase de teste. Embora os pesquisadores não tenham referido, sabe-se que palavras de valência negativa apresentam forte associação semântica entre si (Talmi \& Moschovicht, 2004). Desse modo, acreditamos que os estudos de Maratos et al. (2000) e McNeely (2004) não possibilitam sugerir que as diferenças encontradas entre os índices de falsas memórias de palavras neutras e emocionais sejam decorrentes de um possível efeito da emoção ou da associação semântica.

Outra crítica que levantamos à pesquisa de McNeely et al. (2004) é que eles não utilizaram palavras padronizadas emocionalmente nas dimensões valência e alerta, o que é necessário para garantir que os estímulos utilizados sejam realmente neutros e emocionais. Consideramos que os pesquisadores também deveriam ter empregado palavras de valência positiva, o que possibilitaria a testagem da hipótese evolutiva, que sugere que nossa espécie foi programada, no decorrer de sua história filogenética, a responder de maneira diferenciada a estímulos negativos. Se a hipótese evolutiva for correta, devemos esperar que o índice de falsos reconhecimentos de palavras positivas seja inferior ao de palavras negativas, haja vista que as palavras positivas, por dedução, não são aversivas.

Quanto à metodologia utilizada por Pesta et al. (2001) e Kensinger e Corkin (2004a), esses estudos não utilizaram palavras emocionais na fase de estudo, ou, quando foram acrescentadas, foi em número inferior ao de palavras neutras. A nosso ver, essa abordagem metodológica não é a mais adequada para investigar um possível impacto das emoções na produção de falsas memórias, pois: (1) ao não utilizar palavras emocionais na fase de estudo, não é possível avaliar o efeito da emoção durante essa fase; (2) a ausência de palavras emocionais na fase de estudo, ou a utilização de palavras emocionais em menor número do que as neutras, não permite dissociar o impacto da emoção ao da distintividade conceitual, decorrente da evidente diferença semântica entre as palavras neutras apresentadas na fase de estudo e as palavras emocionais apresentadas somente na fase de teste.

Outro ponto que merece destaque na metodologia empregada nos estudos de Pesta et al. (2001) e Kensinger e Corkin (2004a) é que eles só utilizaram palavras de valência neutra e negativa, excluindo a valência positiva da investigação do impacto da emoção na produção de falsas memórias. Acreditamos que o emprego de palavras caracterizadas por valência positiva poderia auxiliar as investigações. Por exemplo, a hipótese da heurística 
da distintividade poderia ser avaliada pela utilização de itens emocionais positivos. Se ela for adequada, poder-se-ia hipotetizar que o mesmo padrão de resultados encontrado para palavras negativas (e.g., estupro) deveria expressar-se nas taxas de falsas memórias positivas. Isto é, ao ler a palavra emocional positiva na fase de teste (e.g., beijo), o participante pode utilizar a heurística da distintividade ao pensar (e.g.,"se eu tivesse estudado a palavra beijo, com certeza eu lembraria dela"), tornando-se menos propenso a reconhecer falsamente essa palavra no teste de memória.

Consideramos que futuros estudos busquem atentar para esses aspectos apresentados. Sendo assim, sugerimos aos pesquisadores que: (1) controlem a associação semântica entre as palavras apresentadas na fase de estudo e as apresentadas somente na fase de teste, tanto para neutras quanto para emocionais; (2) apresentem materiais emocionais na fase de estudo e na fase de teste, já que a emoção pode impactar o funcionamento da memória desde a codificação das palavras apresentadas na fase de estudo; (3) façam uso de estímulos padronizados quanto à valência e alerta, para certificar que os itens sejam realmente neutros e emocionais, e porque a valência e o alerta podem impactar a produção de falsas memórias de distintas formas; (4) empreguem estímulos classificados como sendo de valência positiva, além dos usuais neutros e negativos, a fim de testar a hipótese evolucionista e a hipótese da heurística da distintividade; (5) igualem o número de itens neutros e emocionais na fase de estudo, a fim de controlar a distintividade conceitual; (6) avaliem a produção de falsas recordações emocionais; (7) manipulem os intervalos entre a fase de estudo e a realização da testagem, considerando que a ação do alerta na fase de consolidação da memória demanda tempo (1 a 24 horas); (8) incluam outras variáveis que reconhecidamente impactam o funcionamento da memória e, conseqüentemente, concorrem com a carga emocional dos estímulos na explicação das falsas memórias emocionais (e.g., concretude dos estímulos - para uma análise do impacto dessa variável na produção de falsas memórias, ver Perez-Mata, Read e Diges (2002); (9) incluam variáveis de personalidade (e.g., o traço neuroticismo - para uma análise do impacto dessa variável na produção de falsas memórias, ver Ávila \& Stein, no prelo); (10) investiguem os mecanismos neurais subjacentes à produção de falsas memórias emocionais; e (11) avaliem a influência do humor na produção de falsas memórias.

Com o objetivo de investigar as falsas memórias em nosso país, Stein, Feix, e Rohenkohl (2006) desenvolveram a versão brasileira do procedimento DRM. Essa versão é composta por 44 listas, sendo 36 construídas a partir de palavras críticas (e.g., música) do estudo de Stadler, Roediger e McDermott (1999). Dessas 36 listas originais havia apenas uma com evidente conteúdo emocional - cuja palavra crítica era raiva. Na versão brasileira foram adicionadas a esse conjunto oito novas listas, cujas palavras críticas representam distintas emoções: quatro listas com palavras críticas 
positivas (alegria, alívio, paixão, satisfação) e quatro listas com palavras críticas negativas (culpa, dor, mágoa, medo). Adicionalmente, Santos, Silveira e Stein (2007) realizaram a normatização afetiva das 44 listas e das 44 respectivas palavras críticas dentro das dimensões valência e alerta. $O$ conhecimento da carga emocional das listas e das palavras críticas permitirá a avaliação, em separado, da influência dessas dimensões na produção de falsas memórias.

Por fim, consideramos que o entendimento da influência das emoções na produção de falsas memórias pode contribuir para a melhor compreensão da relação entre a emoção e o funcionamento da memória. $E$, conseqüentemente, para o desenvolvimento de conhecimentos aplicados, por exemplo, na área da saúde mental e na área jurídica, que levem em consideração o ser humano em sua integralidade.

\title{
The influence of emotions on false memories: a critical review
}

\begin{abstract}
In this article we reviewed studies relating to emotional influence on memory function and specifically, in the occurrence of false memories. False memories are a kind of mnemonic distortion which consist of the recovery of events that never happened. Emotion is being described in terms of dimensions of valence and arousal. Numerous studies have suggested that valence and arousal are beneficial to memory through distinct cognitive processes and neural mechanisms. On the other hand the research about emotional impact on the occurrence of false memories is quite incipient and there is not much consensus with respect to their results. We focus mainly on the methodological discussion of research relating emotion to false recognition. Finally we present some suggestions for future studies, and the current state of research carried out in Brazil.
\end{abstract}

Keywords: Emotion. Memory. False memories. 


\section{L'influence des émotions sur les fausses mémoires: une révision critique}

Résumé:Dans cet article on révise les études rélatifs à l'influence des émotions dans le fonctionnement de la mémoire et, especifiquement, dans la production des fausses mémoires. Les fausses mémoires sont un type de distortion mnemonique qui consistent à la récuperation des événements qui n'ont pas ocurru. L'émotion est décrite à travers les dimensions de valence et alerte. Des nombreux études ont indiqué que la valence et l'alerte aident la mémoire à travers des processus cognitives et des mécanismes neurales distincts. Pourtant, les études sur l'impact de l'émotion sur la production des fausses mémoires sont assez rares et ne sont pas toujours d'accord à propos de leurs résultats. Le point principal en cet article sera la discussion méthodologique des recherches qui relationnent l'émotion à des fausses réconnaissances. Finalement, on présente quelques suggestions d'études futures, aussi que l'état actuel des recherches développées au Brésil.

Mots-clés: Emotion. Mémoire. Fausess memoires.

\section{La influencia de la emoción en los falsos recuerdos: una revisión crítica}

Resumen: En este artículo se revisarán los estudios sobre la influencia de las emociones sobre el funcionamiento de la memoria y, en particular, la producción de falsos recuerdos. Los falsos recuerdos son un tipo de distorsión, que son la memoria acerca de acontecimientos que nunca se produjieron. Las emociones son descritas por las dimensiones de valencia y excitación. Numerosos estudios han sugerido que la valencia y alerta pueden beneficiar la memoria por distintos procesos cognitivos y mecanismos neuronales. Ya la investigación sobre la influencia de las emociones en la producción de falsos recuerdos es todavía incipiente, y difiere en sus resultados. El objetivo principal del presente artículo será el debate metodológico de la investigación que relaciona las emociones con los falsos reconocimientos, y las explicaciones teóricas que se han producido para dar cuenta de este fenómeno. Por último, presentamos algunas sugerencias para futuros estudios, y el estado actual de la investigación desarrollada en Brasil acerca de esta temática.

Palabras-clave: Emoción. Memoria. Falsos recuerdos. 


\section{Referências}

Adolphs, R., Cahill, L., Schul, R., \& Babinsky, R. (1997). Impared declarative memory for emotional material following bilateral amígdala damage in humans. Learning and Memory, 4, 291-300.

Adolphs, R., Tranel, D., \& Denburg, N. L. (2000). Impaired emotional declarative memory following unilateral amygdala damage. Learning and Memory, 7, 180186.

Ávila, L. M., \& Stein, L. M. (no prelo). A influência do traço de personalidade nueuroticismo na suscetibilidade às falsas memórias. Psicologia: Teoria e Pesquisa.

Bentin, S., McCarthy, G., \& Wood, C. C. (1985). Event- related potentials, lexical decision and semantic priming. Electroencephalography and Clinical Neurophysiology, 60(4), 343-355.

Berntsen, D. (2002). Tunnel memories for autobiographical events: Central details are remembered more frequently from shocking than from happy experiences. Memory \& Cognition, 30(7), 1010-1020.

Bradley, M. M., \& Lang, P. J. (1994). Measuring emotion: The self- assessment manikin and semantic differential. Journal of Behavioral Therapy and Experimental Psychiatry, 25(1), 49-59.

Brainerd, C., \& Reyna V. (2005). Theorical explanation of false memories. In C. Brainerd \& V. Reyna, The science of false memory (pp. 59-96). New York: Oxford.

Brainerd, C. J., \& Reyna, V. F. (2002). Fuzzy - Trace and false memory. Current Direction in Psychological Science, 11(5), 164-168.

Bransford, J. D., \& Franks, J. J. (1971). The abstraction of linguistic ideas. Cognitive Psychology, 2, 331-350.

Brown, R., \& Kulik, J. (1977). Flashbulb memories. Cognition, 5, 73-79.

Buchanan, T. W., Denburg, N. L., Tranel, D., \& Adolphs, R. (2001). Verbal and nonverbal emotional memory following unilateral amygdala damage. Learning \& Memory, 8, 326-335.

Burke, A., Heuer, F., \& Reisberg, D. (1992). Remembering emotional events. Memory \& Cognition, 20, 277-290.

Cahill, L., \& McGaugh, J. L. (1998). Mechanisms of emotional arousal and lasting declarative memory. Trends in Neuroscience, 21, 294-293.

Christianson, S. Å., \& Loftus, E. F. (1991). Remembering emotional events: The fate of detailed information. Cognition \& Emotion, 5, 81-108.

Conway, M. A., Anderson, S. J., Larsen, S. F., Donnelly, C. M., McDaniel, M. A., McClelland, A. G., Rawles, R. E., \& Logie, R. H. (1994). The formation of flashbulb memories. Memory \& Cognition, 22, 326- 343.

Craik, F. I. M. (2002). Levels of processing: Past, present, and future? Memory, 10, 305318. 
Dalgleish, T. (2004). The emotional brain. Nature Reviews Neuroscience,5, 583-589.

Damásio, A. R. (1996). O erro de Descartes: emoção, razão e o cérebro humano. São Paulo: Companhia das Letras.

Damásio, A. R. (2000). A second chance for emotion. In R. D. Lane \& L. Nadel (Eds.), Cognitive neurosciences of emotion (pp. 12-23). New York: Oxford University Press.

Deese, J. (1959). On the prediction of occurrence of particular verbal instrusions in immediate recall. Journal of Experimental Psychology, 58, 17-22.

Doerksen, S., \& Shimamura, A. P. (2001). Source memory enhancement for emotional words. Emotion, 1(1), 5- 11.

Dolcos, F., LaBar, K. S., \& Cabeza, R. (2004). Dissociable effects of arousal and valence on prefrontal activity indexing emotional avaluation and subsequent memory: An event- related potential study. Neurolmage, 23(1), 64-74.

Eisen, M. L., Quas, J. A., \& Goodman, G. S. (2002). Memory and suggestibility in the forensic interview. Mahwah, NJ: Lawrence Erlbaum.

Hamann, S. B., Ely, T. D., Grafton, S. T., \& Kilts, C. D. (1999). Amygdala activity related to enhanced memory for pleasant and aversive stimuli. Nature Neuroscience, 2, 289- 293.

Kensinger, E. A. (2004). Remembering emotional experiences: The contribution of valence and arousal. Reviews in the Neurosciences, 15, 241-251.

Kensinger, E. A., \& Corkin, S. (2003). Memory enhancement for emotional words: Are emotional words more vividly remembered than neutral words? Memory \& Cognition, 31(8), 1169-1180.

Kensinger, E. A., \& Corkin, S. (2004a). The effects of emotional content and aging on false memories. Cognitive, Affective, \& Behavioral Neuroscience, 4(1), 1-9.

Kensinger, E. A., \& Corkin, S. (2004b). Two routs to emotional memory: Distinct neural processes for valence and arousal. Proceedings of National Academy of Sciences, 101, 3310-3315.

LaBar, K. S., \& Cabeza, R. (2006). Cognitive neuroscience of emotional memory. Nature Reviews Neuroscience, 7, 54-64.

LaBar, K. S., \& Phelphs, E. A. (1998). Arousal- mediated memory consolidation: Role of the medial temporal lobe in humans. Psychological Science, 9(6), 490-493.

Lang, P. J. (1969). The mechanics of desensitization and the laboratory study of human fear. In C. M. Franks (Ed.), Assessment and status of the behavior therapies. New York: McGraw Hill.

Lang, P. J. (1980). Behavioral treatment and bio-behavioral assessment: Computer applications. In J. B. Sidowski, J. H. Johnson, \& T. A. Williams (Eds.), Technology in mental health care delivery systems (pp. 119-137). Norwood, NJ: Ablex.

Lang, P. J. (1995). The emotion probe: Studies of motivation and attention. American Psychologist, 50(5), 372-385.

Lazarus, R. S. (1991). Emotion and adaptation. New York: Oxford University Press. 
Levine, L. J. (1997). Reconstructing memory for emotions. Journal of Experimental Psychology: General, 126, 165-177.

Levine, L. J., \& Safer, M. A. (2002). Sources of bias in memory for emotions. Current Directions in Psychological Science, 11, 169-173.

Lindsay, D. S. (1994). Contextualizing and clarifying criticisms of memory work in psichoterapy. Consciouness and Cognition, 3, 426-434.

Loftus, E. F., \& Palmer, J. C. (1974). Reconstruction of automobile destruction: An example of interation between language and memory. Journal of Verbal Learning and Verbal Behavior, 13, 585-589.

Loftus, E. F., Miller, D. G., \& Burns, H. J. (1978). Semantic integration of verbal information into a visual memory. Journal of Experimental Psychology: Human Learning and Memory, 4, 19-31.

MacGaugh, J. L. (2004). The amygdala modulates the consolidation of memories of emotiolly arousing experiences. Annual Review of Neuroscience, 27, 1-28.

Maratos, E. J., Allan, K., \& Rugg, M. D. R. (2000). Recognition memory for emotionally negative and neutral words: An ERP study. Neuropsychologia, 38, 1452-1465.

McNeely, H. E., Dywan, J., \& Segalowitz, S. J. (2004). ERP indices of emotionality and semantic cohesiveness during recognition judgments. Psychophysiology, 41, 117129.

Oschner, K. N. (2000). Are affective events richly recollected or simply familiar? The experience and process of recognizing feelings past. Journal of Experimental Psychology: General, 129(2), 242-261.

Paller, K. A., \& Wagner, A. D. (2002). Observing the transformation of experience into memory. Trends Cognitive Sciences, 6, 93-102.

Perez- Mata, M. N., Read, J. D., \& Diges, M. (2002). Effects of divided attention and word concreteness on correct recall and false memory reports. Memory, 10(3), 161-177.

Pesta, B., Murphy, M. D., \& Sanders, R. E. (2001). Are emotionally charged lures immune to false memory? Journal of Experimental Psychology: Learning, Memory, and Cognition, 27(2), 328-338.

Reisberg, D., \& Heuer, F. (2004). Memory for emotional events. In D. Reiberg, P. Hertel (Eds.), Memory and emotion (pp. 3-41). New York: Oxford University Press.

Reyna, V. F., \& Brainerd, C. J. (1995). Fuzzy - Trace Theory: An interim synthesis. Learning and Individual Differences, 7(2), 1-75.

Reyna, V. F., \& Lloyd, F. (1997). Theories of false memory in children and adults. Learning and Individual Differences, 9, 95-124.

Ribeiro, R. L., Pompéia, S., \& Bueno, O. F. A. (2004). Normas brasileiras para o International Affective Picture System (IAPS): comunicação breve. Revista de Psiquiatria do Rio Grande do Sul, 26(2), 190-194.

Roediger, H. L., III, \& McDermott, K. B. (1995). Creating false memories: Remembering words not presented on lists. Journal of Experimental Psychology: Learning, Memory, and Cognition, 21, 803-814. 
Roediger, H. L., III, Watson, J. M., McDermott, K. B., \& Gallo, D. A. (2001). Factors that determine false recall: A multiple regression analysis. Psychonomic Bulletin \& Review, 8, 385-407.

Rubin, D., \& Kozin, M. (1984). Vivid memories. Cognition, 16, 81-95.

Santos, R. F., Silveira, R. A., \& Stein, L. M. (2007). Normas de emocionalidade para a versão brasileira do Procedimento da Lista de Palavras Associadas. Manuscrito submetido para publicação.

Schacter, D. L., Israel, L., \& Racine, C. (1999). Suppressing false recognition in younger and older adults: The distinctriveness heuristic. Journal of Memory and Language, $40,1-24$.

Schacter, D. L., \& Slotnick, S. D. (2004). The cognitive neuroscience of memory distortion. Neuron, 44, 149-160.

Schacter, D. L., Verfaellie, M., \& Anes, M. D. (1997). Illusory memories in amnesic patients: Conceptual and perceptual false recognition. Neuropsychology, 11, 331342.

Schacter, D. L. (2001). The seven sins of memory: How the mind forgets and remembers. Boston: Houghton Mifflin.

Sommers, M. S., \& Lewis, B. P. (1999). Who really lives next door: Creating false memories with phonological neighbors. Journal of Memory and Language, 40, 83-108.

Stein, L. M., \& Pergher, G. K. (2001). Criando falsas memórias em adultos por meio de palavras associadas. Psicologia: Reflexão e Crítica, 14(2), 353-366.

Stein, L. M., Feix, L. F., \& Rohenkohl, G. (2006). Avanços metodológicos no estudo das falsas memórias: Construção e normatização do procedimento de palavras associadas à realidade brasileira. Psicologia: Reflexão e Crítica, 19(2), 196-205.

Talarico, J. M., \& Rubin, D. C. (2003). Confidence not consistency, characterizes flashbulb memories. Psychological Science, 14, 455-461.

Talmi, D., \& Moscovitch, M. (2004). Can semantic relatedness explain the enhancement of memory for emotional words? Memory \& Cognition, 32(5), 742-751.

Watson, J. M., Balota, D. A., \& Sergent- Marshall, S. D. (2001). Semantic, phonological, and hybrid veridical and false memories in healthy older adults and in individuals with Dementia of the Alzheimer type. Neuropsychology, 2, 254-267. 
Renato Favarin dos Santos, Mestre em Psicologia pela Pontifícia Universidade Católica do Rio Grande do Sul, Professor do curso de Psicologia da Faculdade Cathedral, Boa Vista, RR e Psicólogo da Universidade Federal de Roraima. Endereço eletrônico:rfsantos77@yahoo.com.br

Lilian Milnitsky Stein, Docente da Pontifícia Universidade Católica do Rio Grande do Sul e Bolsista produtividade CNPQ. Endereço para correspondência: Pós-Graduação em Psicologia, PUC-RS, Av. Ipiranga,6681, prédio 11, sala 933. CEP 90619-900. Endereço eletrônico: lilian@pucrs.br.

Recebido em: 16/09/2007

Aceito em: 10/09/2008 